OPEN ACCESS

Edited by:

Wei Tao,

Harvard Medical School,

United States

Reviewed by:

Jianxun Ding,

Changchun Institute of Applied

Chemistry (CAS), China

Bingyang Shi,

Macquarie University, Australia

Guoging Pan,

Jiangsu University, China

*Correspondence:

Xiaoding $\mathrm{Xu}$

xuxiaod5@mail.sysu.edu.cn

Herui Yao

yaoherui@126.com Yandan Yao

yaoyand@mail.sysu.edu.cn

Specialty section:

This article was submitted to

Experimental Pharmacology

and Drug Discovery,

a section of the journal

Frontiers in Pharmacology

Received: 28 October 2018 Accepted: 30 November 2018

Published: 14 December 2018

Citation:

Liang S, Zheng J, Wu W, Li Q,

Saw $P E$, Chen J, Xu X, Yao H and Yao Y (2018) A Robust Nanoparticle

Platform for RNA Interference in Macrophages to Suppress Tumor

Cell Migration.

Front. Pharmacol. 9:1465. doi: 10.3389/fphar.2018.01465

\section{A Robust Nanoparticle Platform for RNA Interference in Macrophages to Suppress Tumor Cell Migration}

\author{
Shi Liang 1,2,3, Junmeng Zheng 1,2, Wei Wu 1,2,3, Quan Li $i^{1,2,3}$, Phei Er Saw ${ }^{1,2}$, \\ Jianing Chen ${ }^{1,2,3}$, Xiaoding $\mathrm{Xu}^{1,2 *}$, Herui Yao ${ }^{1,2,3 *}$ and Yandan Yao ${ }^{1,2,3 *}$
}

\begin{abstract}
' Guangdong Provincial Key Laboratory of Malignant Tumor Epigenetics and Gene Regulation, Medical Research Center, Sun Yat-sen Memorial Hospital, Sun Yat-sen University, Guangzhou, China, ${ }^{2}$ RNA Biomedical Institute, Sun Yat-sen Memorial Hospital, Sun Yat-sen University, Guangzhou, China, ${ }^{3}$ Breast Tumor Center, Sun Yat-sen Memorial Hospital, Sun Yat-sen University, Guangzhou, China
\end{abstract}

Macrophages are one of the most abundant immune cells in the solid tumor and their increased density is associated with the specific pathological features of cancers, including invasiveness, metastasis, immunosuppression, neovascularization, and poor response to therapy. Therefore, reprogramming macrophage behavior is emerging as a promising therapeutic modality for cancer treatment. RNA interference (RNAi) technology is one of the powerful strategies for the regulation of macrophage activities by silencing specific genes. However, as polyanionic biomacromolecules, RNAi therapeutics such as small interfering RNA (siRNA) cannot readily cross cell membrane and thus specific delivery vehicles are required to facilitate the cytosolic siRNA delivery. Herein, we developed a robust nanoparticle (NP) platform for efficient siRNA delivery and gene silencing in macrophages. This NP platform is composed of biodegradable poly (ethylene glycol)- $b$-poly ( $\varepsilon$-caprolactone) (PEG- $b$-PCL), poly ( $\varepsilon$-caprolactone)- $b$-poly (2-aminoethyl ethylene phosphate) (PCL-b-PPEEA), and PCL homopolymer. We chose CC-chemokine ligand 18 (CCL-18) as a proof of concept therapeutic target and our results demonstrate that the CCL-18 silencing in macrophages can significantly inhibit the migration of breast cancer cells. The successful regulation of the macrophage behavior demonstrated herein shows great potential as an effective strategy for cancer therapy.

Keywords: macrophages, RNAi, nanoparticle, cancer therapy, siRNA delivery

\section{INTRODUCTION}

Macrophages are important cells of immune system with two major phenotypes, i.e., proinflammatory phenotype (M1) and anti-inflammatory phenotype (M2) (Mantovani et al., 2008; Noy and Pollard, 2014; Ostuni et al., 2015). In solid tumors, tumor-associated macrophages (TAMs) are one of the most abundant cell types (up to $50 \%$ of the tumor mass) and are present at all 
stages of tumor progression (Caillou et al., 2011; Ginhoux et al., 2015; Parayath et al., 2018). Numerous clinical and epidemiological studies have demonstrated that TAMs are primary M2-like macrophages (Gordon and Martinez, 2010; Sica and Mantovani, 2012; Bronte and Murray, 2015) and their increased density is associated with the specific pathological features of cancers, including invasiveness, metastasis, immunosuppression, neovascularization, and poor response to therapy (Qian and Pollard, 2010; McAllister and Weinberg, 2014; Bronte and Murray, 2015). Therefore, macrophages represent an important therapeutic target and strategies that can effectively regulate undesirable macrophage activities are always pursued for future cancer therapy.

One of the promising strategies for the regulation of macrophage activities is using RNA interference (RNAi) technology to silence specific genes (Aouadi et al., 2009; Kortylewski et al., 2009; Yu et al., 2013). Since its discovery in 1998, RNAi technology has demonstrated significant potential for disease treatment by silencing the expression of target gene(s), especially those encoding "undruggable" proteins (Melnikova, 2007; Burnett and Rossi, 2012; Kanasty et al., 2013; Zuckerman and Davis, 2015). The key challenge is the safe and effective delivery of RNAi therapeutics such as small interfering RNA (siRNA) to aberrant macrophages (e.g., TAMs). Due to its polyanionic and macromolecular characteristics, naked siRNA cannot readily cross the cell membrane and thus requires specific delivery vehicles to facilitate its intracellular uptake and cytosolic delivery for bioactivity (Whitehead et al., 2009; Zhang et al., 2012; Shi et al., 2017; Xu et al., 2017b). Over the past decade, nanoparticles (NPs), which present the advantage of preferential and selective accumulation at tumor sites via the enhanced permeation and retention (EPR) effect, have been widely used for cancer treatment (Chen et al., 2017; Zeng et al., 2017; Liu et al., 2018; Xiao et al., 2018). Up to now, numerous innovative NPs have been developed to enhance the siRNA delivery efficacy (Tseng et al., 2009; Xu et al., 2016, 2017a; Cheng et al., 2017; Saw et al., 2018). However, a substantial number of these NPs are designed to directly target tumor cells. At present, modest effort has been made to develop RNAi NPs for the modulation of undesirable macrophage activities.

Herein, we developed a robust RNAi NP platform for the efficient regulation of macrophage activities. This NP platform is composed of biodegradable poly ( $\varepsilon$-caprolactone)- $b$ poly (2-aminoethyl ethylene phosphate) (PCL- $b$-PPEEA), poly (ethylene glycol)- $b$-poly ( $\varepsilon$-caprolactone) (PEG- $b$-PCL), and PCL homopolymer (Figure 1). Through optimizing the NP size by adjusting the formulation, we demonstrated that larger size NPs can deliver siRNA and silence target gene in macrophages with higher efficacy. As a proof-of-concept, we chose CC-chemokine ligand 18 (CCL-18) as a therapeutic target and evaluated the influence of CCL-18 silencing on the macrophage activities. CCL-18 is a key factor secreted by TAMs to induce cancer cell epithelial-mesenchymal transition (EMT), enhance breast cancer metastasis, and reduce patient survival (Chen et al., 2011; Su et al., 2014; Nie et al., 2017). Our results show that the optimal NP platform can efficiently silence the CCL-18 expression in macrophages, leading to significant inhibition of breast cancer cell migration (Figure 1).

\section{MATERIALS AND METHODS}

\section{Materials}

Methoxyl-poly (ethylene glycol) (Meo-PEG $114-\mathrm{OH}, M_{n}=5000$ ), phorbol myristate acetate (PMA), N-2-hydroxyethylpiperazine$N^{\prime}$-2-ethanesulfonic acid buffered saline (HEPES), stannous octoate [Sn $\left.(\mathrm{Oct})_{2}\right]$, RNase A, and heparin sulfate were acquired from Sigma-Aldrich and used directly. $\varepsilon$-Caprolactone (CL) was provided by Sigma-Aldrich and distilled before use. The poly ( $\varepsilon$-caprolactone) homopolymer with 34 repeating units $\left(\mathrm{PCL}_{34}\right)$ and polydispersity of 1.21 was synthesized according to the previous report (Wang et al., 2006). The block copolymers, methoxyl-poly (ethylene glycol)-block-poly ( $\varepsilon$-caprolactone) $\left(\mathrm{mPEG}_{114}-b-\mathrm{PCL}_{41}\right)$ and poly ( $\varepsilon$-caprolactone)-block-poly (2-aminoethyl ethylene phosphate) $\left(\mathrm{PCL}_{25}-b\right.$-PPEEA 17$)$, were synthesized according to our previous reports (Wang et al., 2013; Liang et al., 2015). The degree of polymerization of each repeating unit was calculated based on proton nuclear magnetic resonance $\left({ }^{1} \mathrm{HNMR}\right)$ analysis. Dulbecco's modified Eagle's medium (DMEM), 3-[4,5-dimethylthiazol2-yl]-2,5-diphenyltetrazoliumbromide (MTT), fetal bovine serum (FBS), and trypsin were purchased from Gibco BRL. Lipofectamine 2000 (Lipo) RNAi MAX transfection kit and DAPI were provided by Invitrogen Corp. Fluorescent dye (Cy5) labeled CCL-18 siRNA (Cy5-siCCL-18) and negative control siRNA (siNC) were acquired from Suzhou Ribo Life Science Co., The siRNA sequences are as follows: siNC, 5'-TTG GGA AAA TGA GTG GTT dTdT-3' (sense) and $5^{\prime}$-AAC CAC TCA ACT TTT TCC CAA dTdT-3' (antisense); siCCL-18, 5'-ACA AGU UGG UAC CAA CAA ATT-3' (sense) and 5'-UUU GUU GGU ACC AAC UUG UGC -3' (antisense). The fluorescent dye was labeled at the $5^{\prime}$-end of the sense strand of siCCL-18. All other organic solvents or reagents were analytical grade and used without further purification.

\section{Methods \\ Preparation and Characterizations of Nanoparticles (NPs)}

The NPs with different sizes were prepared by using the classic nanoprecipitation method. The polymers, mPEG- $b$-PCL (10 mg), PCL- $b$-PPEEA (10 mg), and PCL (50 mg) were, respectively, dissolved in $1 \mathrm{~mL}$ of acetonitrile and methyl alcohol (v/v, 1:1). Subsequently, mPEG- $b$-PCL and PCL- $b$ PPEEA were mixed in a molar ratio of $1.5: 1$ and then added dropwise to 10 -fold volume of deionized water which was under vigorously stirring. After stirring for another $20 \mathrm{~min}$, the NP suspension was transferred into a rotary evaporator to remove the organic solvent. The final NP suspension was dispersed in deionized water at a concentration of $1 \mathrm{mg} / \mathrm{mL}$. To adjust the NP size, different amount of PCL was mixed with the mixture of mPEG- $b$-PCL and PCL$b$-PPEEA (molar ratio, 1.5:1) and the resulting NPs were 


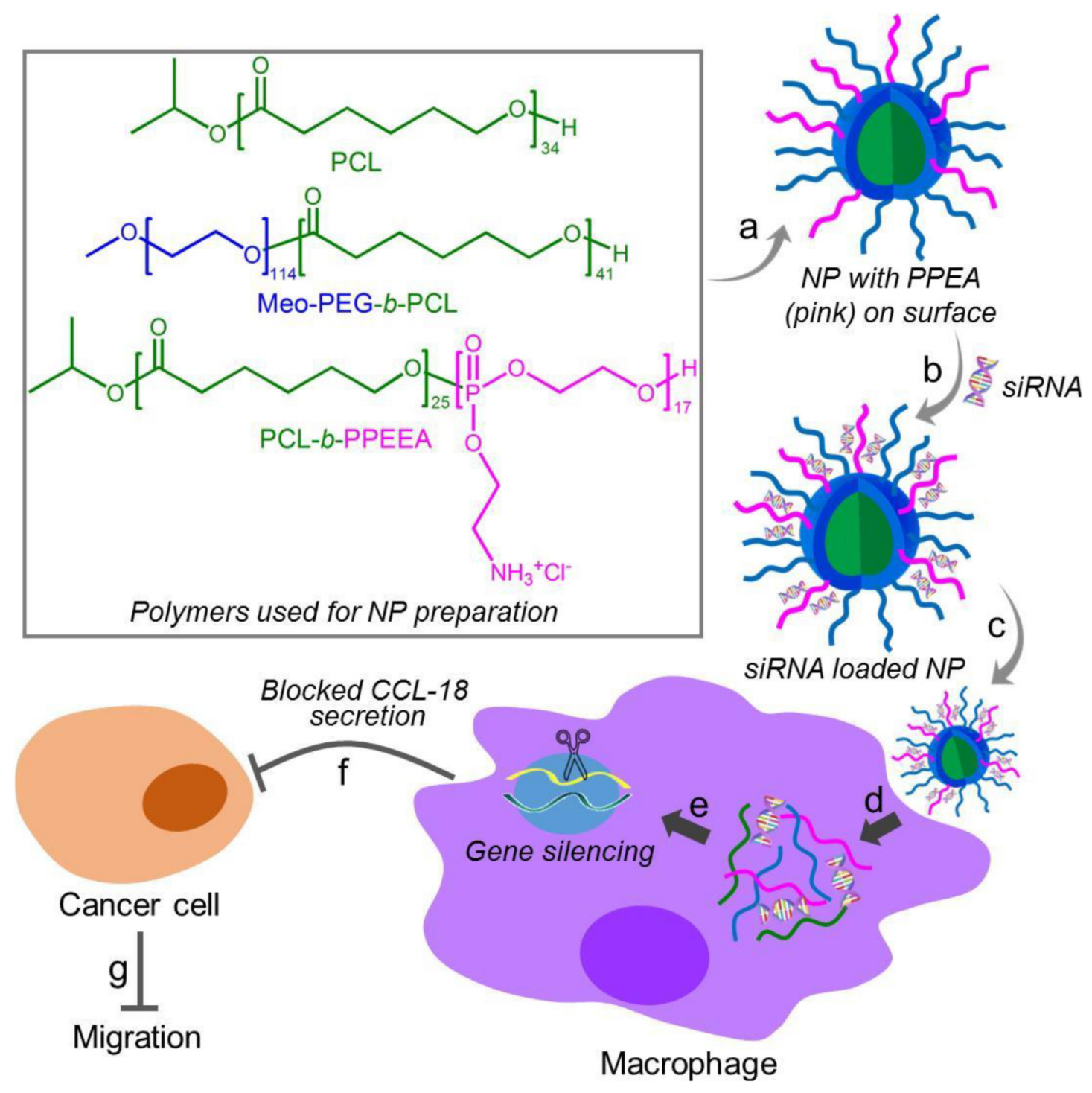

FIGURE 1 | Chemical structures of the polymers (PCL, Meo-PEG-b-PCL, and PCL-b-PPEEA) and schematic illustration of the self-assembly of the polymers into nanoparticles (NPs) for small interfering RNA (siRNA) delivery and CCL-18 silencing in macrophages to inhibit breast cancer migration. The amphiphilic Meo-PEG-b-PCL and PCL-b-PPEEA can spontaneously self-assemble into NPs with hydrophobic PCL chains embedded in the cores and hydrophilic PEG and PPEEA chains positioned on the surface (a). After siCCL-18 loading via the electrostatic interaction (b) and then internalized by macrophages (c,d), the siCCL-18 can knock down CCL-18 expression (e) and thus CCL-18 secretion from the macrophages would be blocked (f), leading to the inhibition of tumor migration (g).

prepared according to the same method described above. The size distribution and zeta potential of the NPs were examined by dynamic light scattering (DLS, Malvern Instruments Corporation). The morphology of the NPs was observed by transmission electron microscopy (TEM, Tecnai $\mathrm{G}^{2}$ Spirit BioTWIN).

\section{Gel Retardation Assay}

The NPs prepared above were mixed with the siCCL-18 aqueous solution $(20 \mathrm{mM})$ at different $\mathrm{N} / \mathrm{P}$ ratios. After incubating at room temperature for $20 \mathrm{~min}$, the formed NP/siCCL-18 complexes were electrophoresed on a $1 \%$ agarose gel at $120 \mathrm{mV}$ for $10 \mathrm{~min}$ in $\mathrm{pH} 8.3$
Tris/borate/EDTA buffer ( $89 \mathrm{mM}$ Tris, $89 \mathrm{mM}$ boric acid, 2 mMEDTA). The siRNA bands were visualized with ethidium bromide staining under a UV transilluminator at a wavelength of $365 \mathrm{~nm}$. Naked siCCL-18 was used as control.

\section{In vitro siRNA Release}

The NP/Cy5-siCCL-18 complexes at an N/P ratio of 10:1 were prepared according the same method described above and then suspended in $\mathrm{pH}$ 7.4 PBS solution at a siRNA concentration of 200 nM. Subsequently, the siRNA loaded NP suspension was transferred to a dialysis device (MWCO $100 \mathrm{kDa}$ ) that was immersed in $\mathrm{pH} 7.4 \mathrm{PBS}$ solution at $37^{\circ} \mathrm{C}$. At a predetermined 

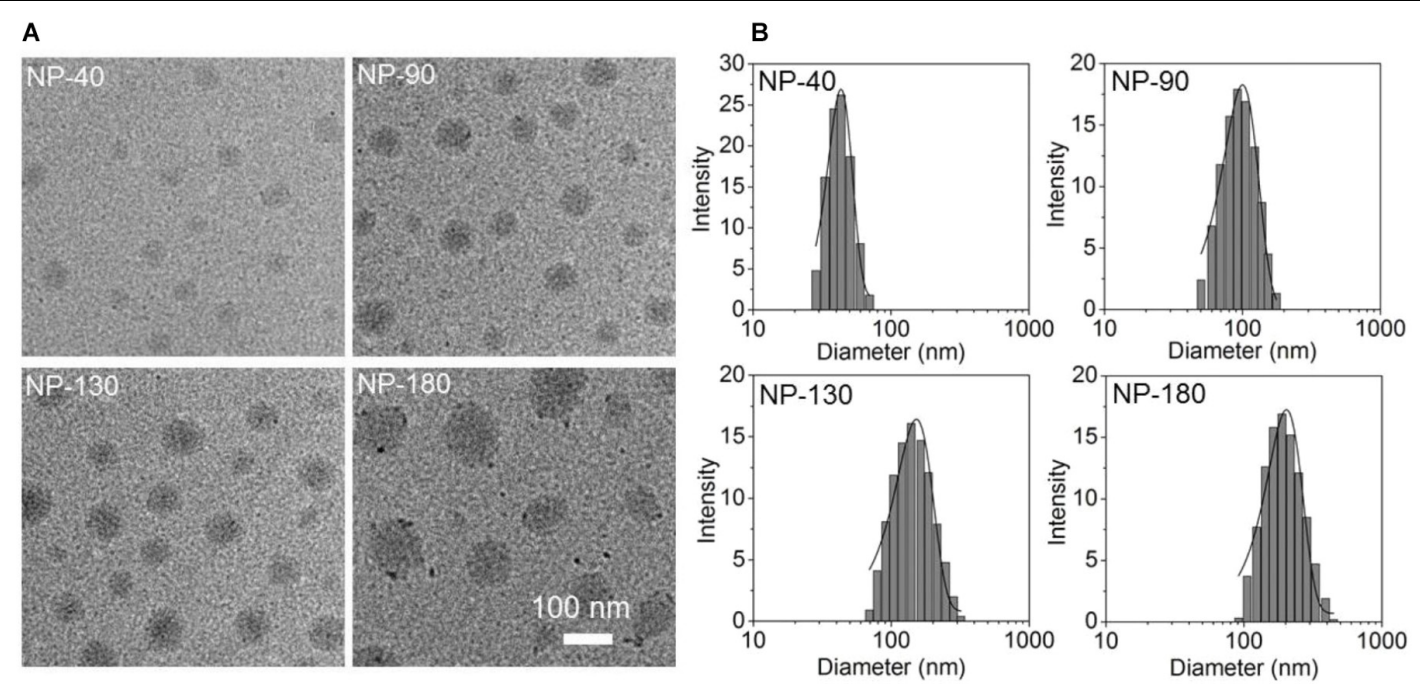

FIGURE 2 | (A) Transmission electron microscopic (TEM) images and (B) size distribution of the NP-40, NP-90, NP-130, and NP-180.

interval, $5 \mu \mathrm{L}$ of the NP suspension was withdrawn and mixed with 20-fold DMSO. The fluorescence intensity of Cy5-siCCL-18 was determined by a microplate reader.

\section{Evaluation of the Stability of NP/siRNA Complexes}

The NP/siCCL-18 complexes were prepared at an N/P ratio of 10:1 and then dispersed in DMEM containing 10\% FBS at $37^{\circ} \mathrm{C}$. At a predetermined interval, the size distribution of the $\mathrm{NP} /$ siCCL-18 complexes was examined by DLS.

\section{Cell Culture}

THP-1 cells (human monocytic leukemia cell line) and human breast cancer cells (MDA-MB-231 cell line) were obtained from the American Type Culture Collection (ATCC) and incubated in DMEM medium with $10 \% \mathrm{FBS}$ at $37^{\circ} \mathrm{C}$ in a humidified atmosphere containing $5 \% \mathrm{CO}_{2}$.

\section{Construction of M2-Like Macrophages}

THP-1 cells were incubated in DMEM medium containing 10\% FBS and PMA (10 ng/mL) at for $8 \mathrm{~h}$. Subsequently, the medium was replaced by fresh DMEM medium containing interleukin-4 (IL-4, $20 \mathrm{ng} / \mathrm{mL}$ ). After $48 \mathrm{~h}$ incubation, the cells will differentiate into M2-like macrophages and the mRNA level of CCL-18 was examined using quantitative reverse transcription real-time PCR (qRT-PCR).

\section{Confocal Laser-Scanning Microscope (CLSM)}

After successful construction of the M2-like macrophages, the cells (50,000 cells) were seeded in round disks and incubated in $2 \mathrm{~mL}$ of DMEM medium containing 10\% FBS. After $24 \mathrm{~h}$ incubation, the medium was replaced and the NP/Cy5siCCL-18 complexes were added to the disks at a siRNA concentration of $200 \mathrm{nM}$. After $4 \mathrm{~h}$ incubation, the medium was removed and the cells were washed with $\mathrm{pH} 7.4$ PBS solution thrice. Finally, the nuclei were stained with DAPI and the cells were viewed under a Carl Zeiss LSM 710 CLSM.

\section{Flow Cytometry}

The M2-like macrophages were seeded in six-well plate $(50,000$ cells per well) and incubated in $2 \mathrm{~mL}$ of DMEM medium containing $10 \%$ FBS. After $24 \mathrm{~h}$ incubation, the medium was replaced and the NP/Cy5-siCCL-18 complexes were added at a siRNA concentration of $200 \mathrm{nM}$. After $4 \mathrm{~h}$ incubation, the medium was removed and the cells were washed with pH 7.4 PBS solution thrice. Finally, the cells were collected for flow cytometry analysis using a BD FACSCalibur flow cytometer.

\section{Cytotoxicity}

The cytotoxicity of the NPs was evaluated by using MTT assay. The M2-like macrophages were seeded in 96-well plates at 5,000 cells per well and incubated in $100 \mu \mathrm{L}$ of DMEM medium containing $10 \%$ FBS. After $24 \mathrm{~h}$ incubation, the medium was removed and different amounts of the NPs suspended in the culture medium were added. After $48 \mathrm{~h}$ incubation, the medium was removed and the cell viability was examined using the MTT assay according to the manufacturer's protocol.

\section{CCL-18 Silencing}

The M2-like macrophages were seeded in six-well plate $(50,000$ cells per well) and incubated in $2 \mathrm{~mL}$ of DMEM medium containing $10 \%$ FBS. After $24 \mathrm{~h}$ incubation, the medium was replaced and the NP/siCCL-18 complexes were added at different siRNA concentrations. After $48 \mathrm{~h}$ incubation, the medium was removed and the cells were washed with $\mathrm{pH}$ 7.4 PBS solution thrice. The intracellular mRNA was isolated and the mRNA level of CCL-18 was examined using qRTPCR.

\section{Inhibition of Migration}

MDA-MB-231 cells (50,000 cells) were seeded in round disks and incubated in $2 \mathrm{~mL}$ of DMEM medium containing 10\% FBS. After $24 \mathrm{~h}$ incubation, the cells in the predesigned area of the disks were removed using tips. After washing the cells with PBS thrice, 
A
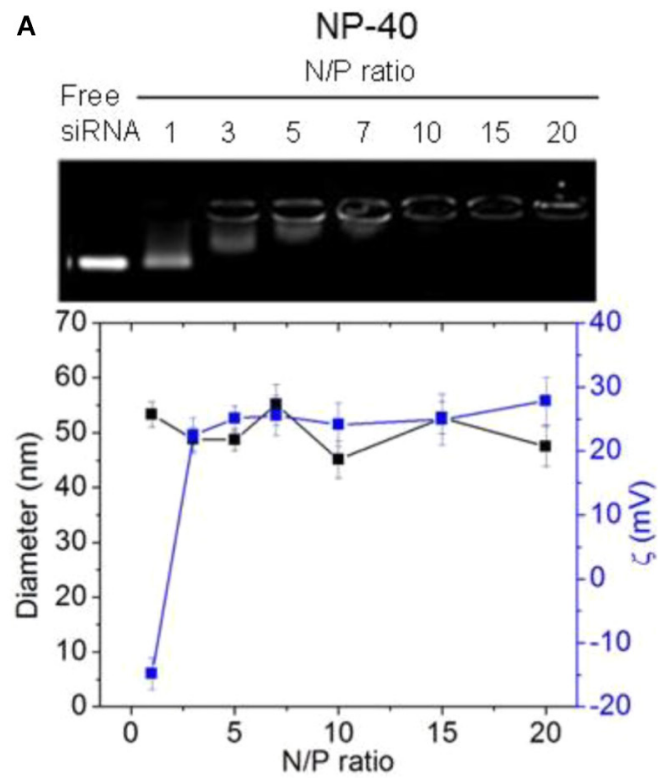

C
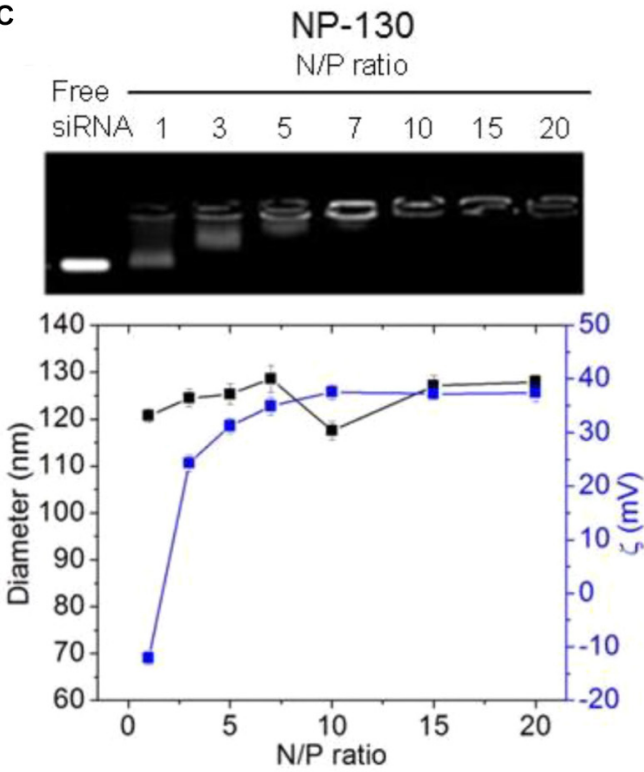

B
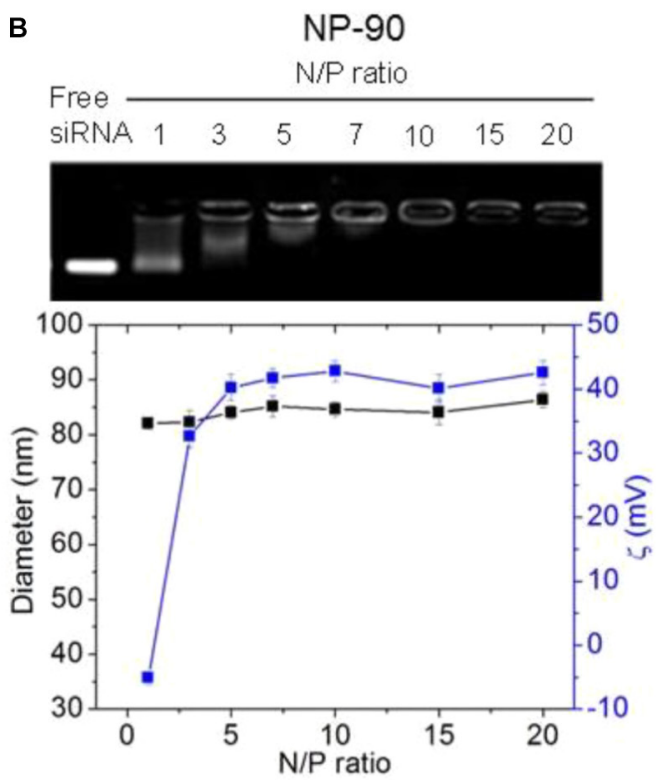

D
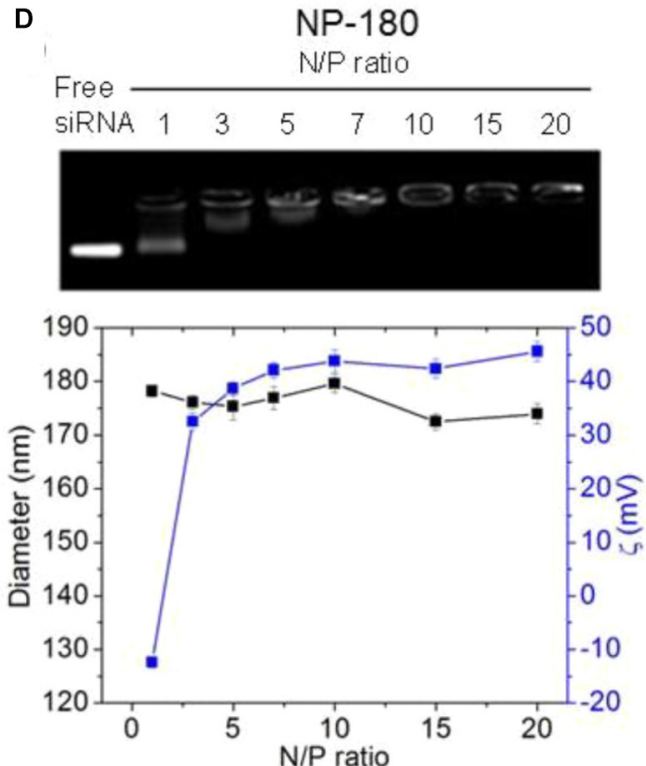

FIGURE 3 | Gel retardation analysis, size, and zeta potential of the complexes formed between siCCL-18 and the NP-40 (A), NP-90 (B), NP-130 (C), or NP-180 (D)

the cells were incubated with the conditioned medium from the macrophages, in which the CCL-18 has been silenced by the NP$180 /$ siCCL-18 at a siRNA concentration of $400 \mathrm{nM}$. After $48 \mathrm{~h}$ incubation, the medium was removed and the cells were viewed under optical microscope after washing with PBS thrice.

\section{RESULTS AND DISCUSSION}

\section{Preparation and Characterizations of Nanoplatform}

Starting from the commercial available mPEG-OH and $\varepsilon$-CL, we employed ring-opening polymerization (ROP) to synthesize the mPEG- $b$-PCL and PCL homopolymer (Sun et al., 2008). The amphiphilic polymer PCL- $b$-PPEEA was also prepared by ROP method (Sun et al., 2008), in which the cationic PPEEA segment was used to complex siRNA via electrostatic interaction. By mixing these three polymers in acetonitrile and methyl alcohol (v/v, 50:50) followed by the addition to deionized water, well-defined NPs can be formed with spherical morphology (Figure 2A). In this self-assembly system, the amphiphilic PEG- $b$-PCL and PCL- $b$-PPEEA can spontaneously self-assemble into NPs with hydrophobic PCL chains embedded in the cores and hydrophilic PEG and PPEEA chains positioned on the surface that can, respectively, stabilize the NPs and complex 


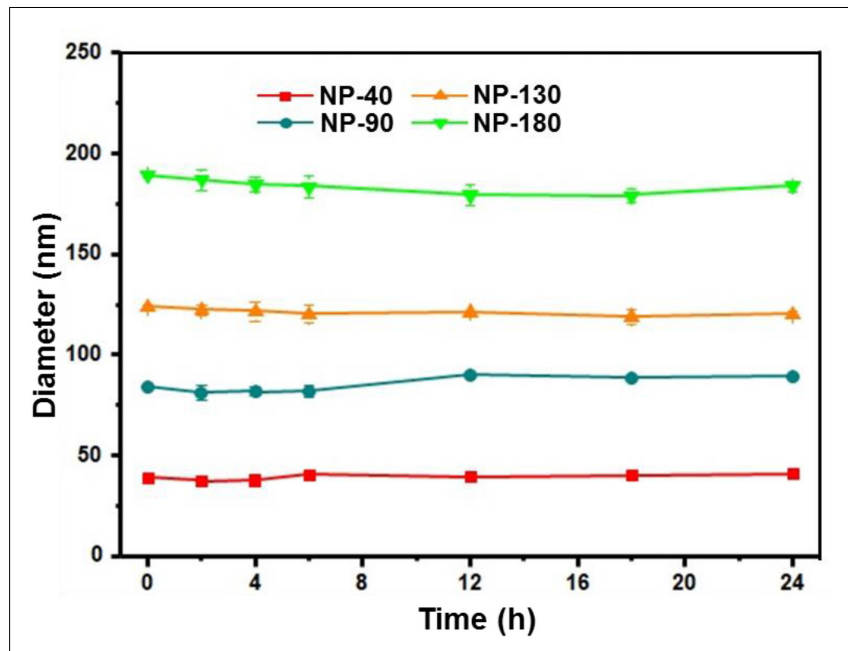

FIGURE 4 | Size of the complexes formed between siCCL-18 and NP-40, NP-90, NP-130, or NP-180 incubated in DMEM medium containing 10\% FBS for different times. negatively charged siRNA. Moreover, with the increasing feed amount of PCL homopolymer, the average size of the resulting NPs increases from $\sim 40$ to $180 \mathrm{~nm}$ (Figure 2B). The possible reason is that the increased percentage of PCL homopolymer in the NPs induces the size increase of their hydrophobic inner cores (Mao et al., 2014; Liang et al., 2015). In this work, we prepared four types of NPs with the size of 40,90, 130, and $180 \mathrm{~nm}$ (respectively, denoted NP-40, NP-90, NP-130, and NP-180) to evaluate their ability to deliver CCL-18 siRNA (siCCL-18) for gene silencing in macrophages.

We next used gel retardation assay to evaluate the siRNA loading capacity of the NPs. As shown in Figure 3, due to the presence of cationic PPEEA segment on the surface, all the NPs can effectively load siCCL18 at an N/P ratio of 10:1, without apparent size change after siCCL18 loading. Furthermore, all the siCCL18 loaded NPs show good stability in 10\% FBScontaining cell culture medium (Figure 4). More importantly, in comparison with the naked siCCL18, the use of the NPs can protect the siCCL18 from degradation by RNase. As shown in Figure $\mathbf{5 A}$, with the protection by the NPs, the addition of RNase does not induce the siRNA degradation and the loaded siRNA can still bind with the NPs even under electric field. In contrast, without the NP protection, the naked siRNA has been degraded by the RNase and thus no siRNA band can be observed in the gel retardation assay experiment. All these results demonstrate the NP platform developed in this work shows a strong ability to load and protect the siCCL-18, which would thus ensure its bioactivity when used for regulation of macrophage activities. Notably, since we only varied the feed amount of hydrophobic PCL homopolymer to adjust the NP size, while the other two polymers (Meo-PEG-b-PCL and PCL$b$-PPEEA) remain constant in the NP formulations, all the NPs showed the similar ability to load and release the siRNA (Figure 5B).

\section{Evaluation of CCL-18 Silencing}

After validation of the siRNA loading ability of the NPs, we next examined whether these NPs can deliver siRNA to macrophages for gene silencing. THP-1, a human monocytic leukemic cell line, was used to construct M2type macrophage-like cells through treatment with PMA and interleukin-4 (IL-4) (Liang et al., 2015). As shown in Figure 6A, the high expression of CCL-18, a well-known chemokine generated by M2-type macrophages (Chen et al., 2011), demonstrated the success of THP-1 cells differentiation from monocytes to M2-type macrophages (denoted THP-1originated macrophages). With this encouraging result, we subsequently encapsulated dye-labeled siCCL-18 (Cy5-siCCL18) into the NPs and investigated their cellular uptake by the differentiated THP-1 macrophages obtained above. From the flow cytometry (FACS) analysis shown in Figure $\mathbf{6 B}$, the THP-1-originated macrophages show higher uptake of the Cy5-siCCL18 loaded NPs compared to the naked siRNA. The intracellular mean fluorescence intensity (MFI) is at least 1.3-fold stronger than that of the macrophages treated with the naked siRNA. Among these NP formulations, the uptake of NP-180 is highest and the possible reason is that macrophages are apt to internalize foreign materials with large size (Tabata and Ikada, 1988; Champion et al., 2008). Figure 6C shows the fluorescent images of macrophages incubated with the Cy5-siCCL-18 loaded NP-180. Similar as the results of FACS analysis, the THP-1-originated macrophages show strong ability to internalize the siRNA loaded NP-180 as seen with bright red fluorescence and these NPs are mainly dispersed in the cytoplasm where siRNA functions (Whitehead et al., 2009). Although the THP-1originated macrophages show higher cellular uptake of the NP-180, these NPs do not induce apparent cytotoxicity (Figure 7A).

Based on the results of FACS analysis and toxicity assay, the NP-180 shows higher uptake by the THP-1-originated macrophages with negligible toxicity. Therefore, we chose this $\mathrm{NP}$ platform as siCCL-18 delivery tool to examine its gene silencing efficacy in macrophages. As shown in Figure $7 \mathbf{B}$, the NP-180 can indeed transport siCCL-18 into the THP-1originated macrophages and thereby down-regulate CCL-18 expression. Compared to the macrophages without any treatment (Control), the mRNA level of CCL-18 is down-regulated by $20 \%$ at a siCCL-18 concentration of $100 \mathrm{nM}$ and more than $70 \%$ of CCL-18 is suppressed at a siCCL-18 concentration of $400 \mathrm{nM}$.

\section{Evaluation of the Inhibition of Migration}

It is known that CCL-18 is an important factor secreted by TAMs that can enhance breast cancer metastasis and therefore reduce patient survival (Chen et al., 2011; Su et al., 2014). Previous reports demonstrate that CCL-18 released by TAMs in breast cancer promotes the invasiveness of cancer cells by triggering integrin clustering and enhancing their adherence to extracellular matrix and silencing the CCL-18 expression can inhibit the consistent activation 

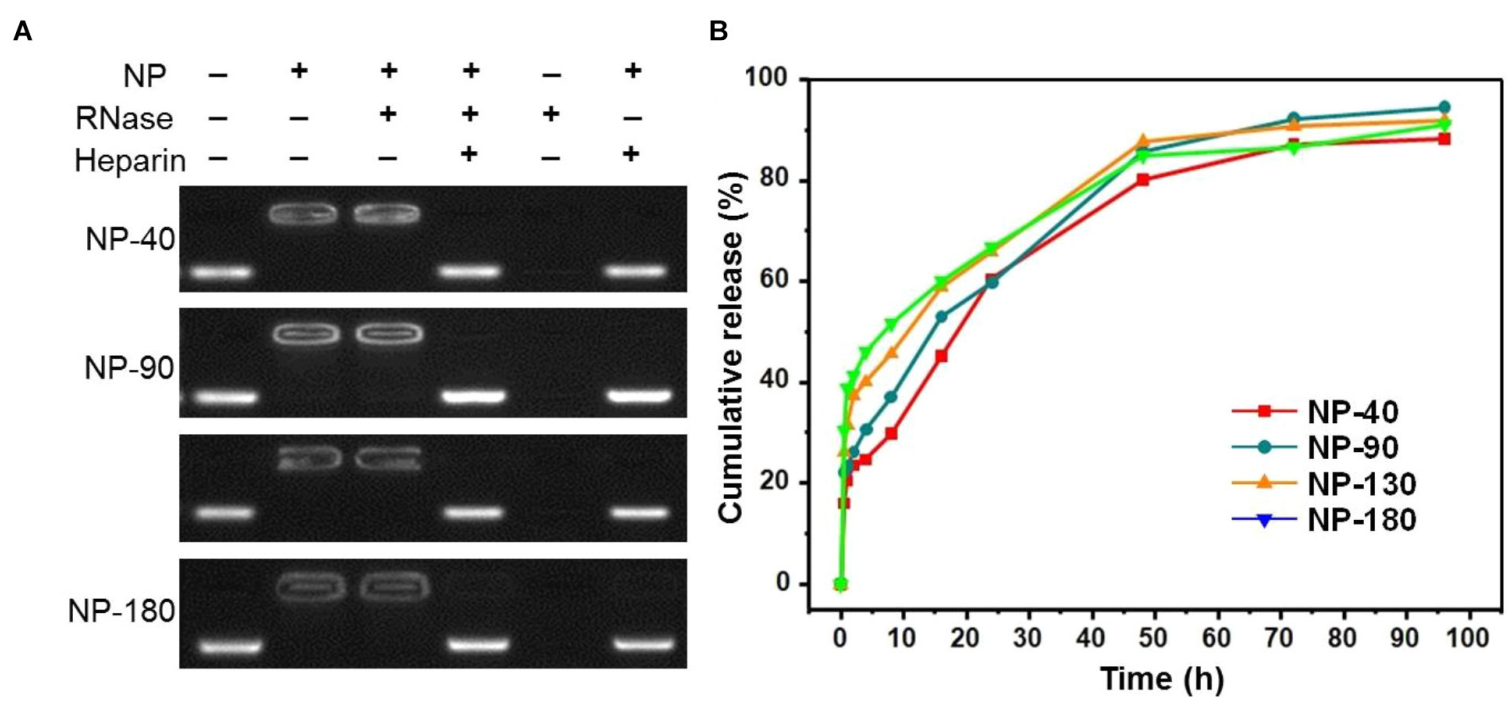

FIGURE 5 | (A) Gel retardation assay of the siRNA loading capacity of the NP-40, NP-90, NP-130, and NP-180; (B) Cumulative siRNA release profile of the complexes formed between Cy5-siCCL-18 and the NP-40, NP-90, NP-130, or NP-180.

A

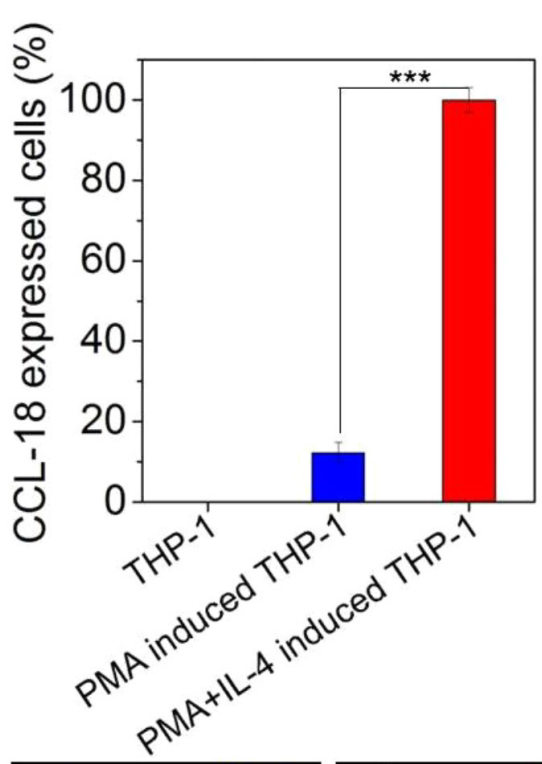

C

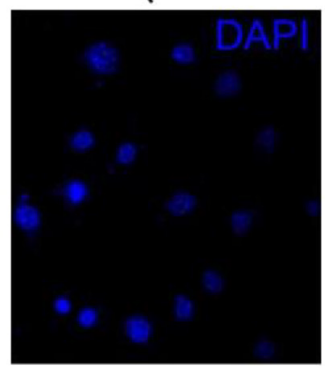

B

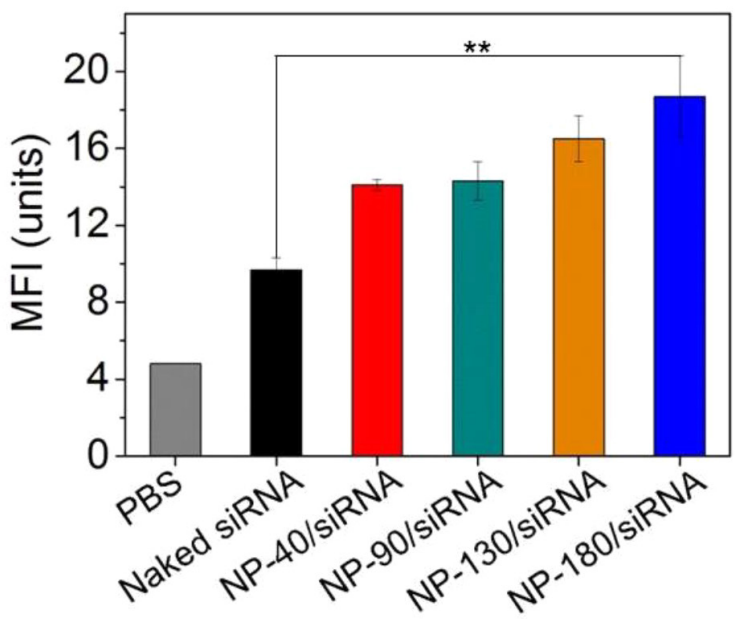

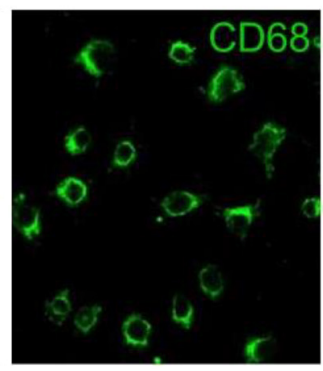
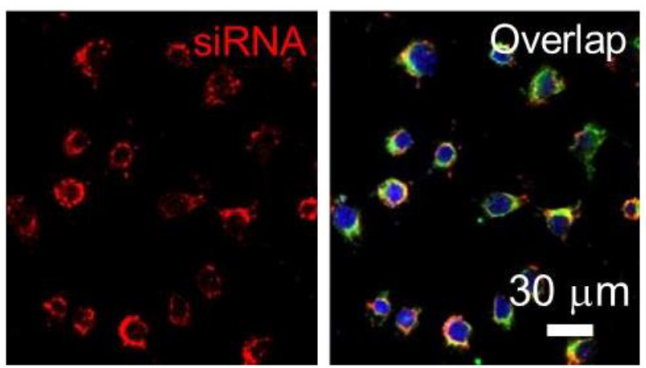

FIGURE 6 | (A) The mRNA level of CCL-18 in the THP-1 cells treated with PMA and IL-4. (B) MFI determined by FACS analysis of the THP-1-originated macrophages incubated with the Cy5-siCCL-18 loaded NPs for $4 \mathrm{~h}$ at a siRNA concentration of $200 \mathrm{nM}$. (C) Fluorescent images of the macrophages incubated with the Cy5-siCCL-18 loaded NP-180 for $4 \mathrm{~h}$ at a siRNA concentration of $200 \mathrm{nM}$. The nuclei and macrophages were staining with DAPI as blue fluorescence and anti-CD68 as green fluorescence, respectively. ${ }^{* *} P<0.01$; ${ }^{* * *} P<0.001$. 
A

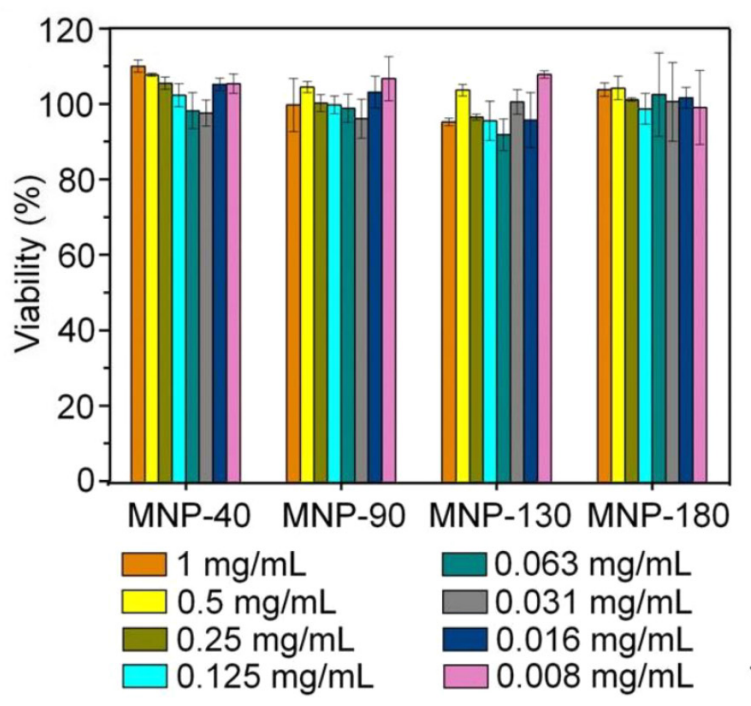

B

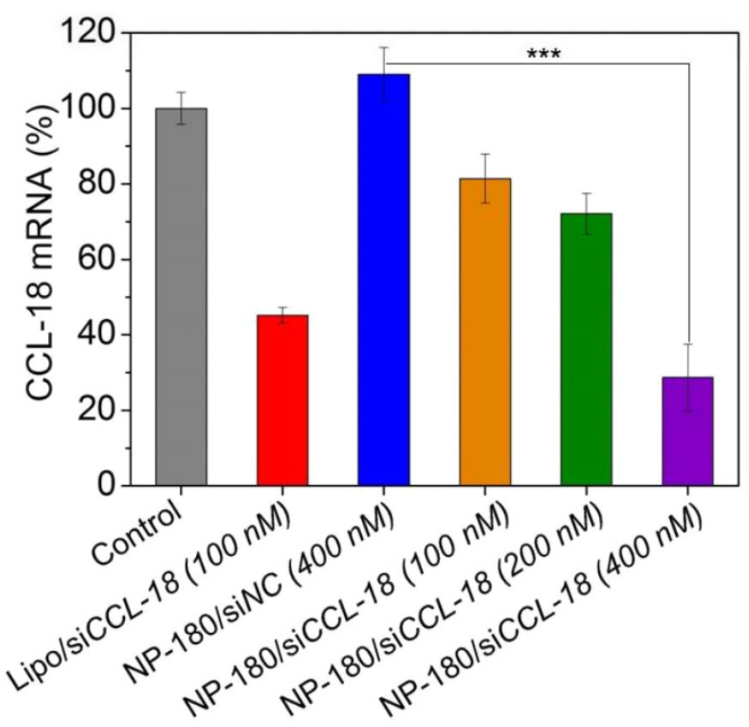

C

Conditioned medium from macrophages

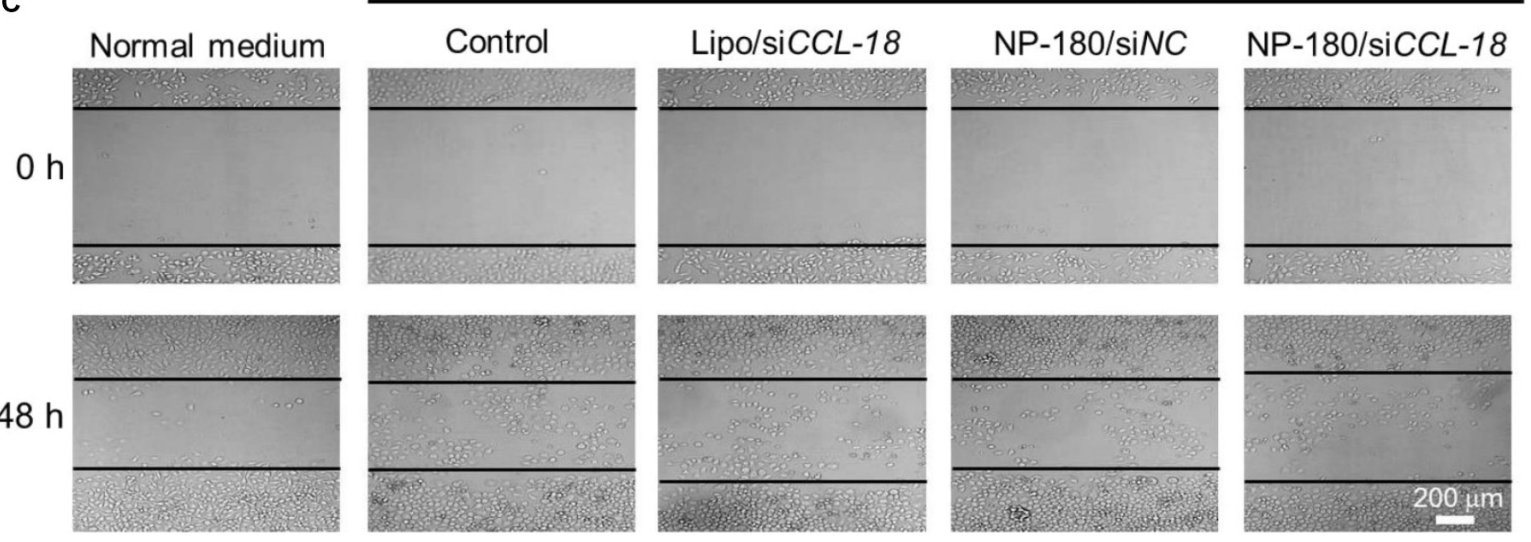

FIGURE 7 | (A) Cell viability of the macrophages incubated with the siCCL-18 loaded NPs at various concentrations for $48 \mathrm{~h}$. (B) The mRNA level of CCL-18 in the macrophages treated by Lipo/siCCL-18 complexes (Lipo/siCCL-18), negative control siRNA loaded NP-180 (NP-180/siNC), and siCCL-18 loaded NP-180 (NP-180/siCCL-18). (C) Optical images of MDA-MB-231 cells incubated with the conditioned medium from the macrophages that treated with Lipo/siCCL-18, NP-180/siNC, and NP-180/siCCL-18 at a siRNA concentration of $400 \mathrm{nM} .{ }^{* * *} P<0.001$.

of downstream signal pathways to prevent the migration of breast cancer (Chen et al., 2011). Therefore, we finally simulated tumor microenvironment in vitro by incubating breast cancer cell line (MDA-MB-231) with the medium that was obtained from THP-1-originated macrophage (denoted conditioned medium), and examined the influence of CCL18 silencing on the behavior of MDA-MB-231 cells. As shown Figure 7C, due to presence of secreted CCL-18 by macrophages, the addition of conditioned medium to MDAMB-231 cells can enhance their migration compared to the cells incubated in normal medium. In contrast, after using siCCL-18 loaded NP-180 to suppress CCL-18 expression in macrophages, the migration of MDA-MB-231 cells is significantly inhibited when incubated with the conditioned medium. This result is consistent with our previous reports (Chen et al., 2011; Su et al., 2014; Nie et al., 2017), and highlights the importance of CCL-18 to the breast cancer cell migration.

\section{CONCLUSION}

We have developed a robust RNAi NP platform for efficient gene silencing in M2-type macrophages. Through varying the percentage of hydrophobic PCL homopolymer in the NP formulation, we successfully constructed four types of NPs with different sizes and systemically evaluated their siRNA loading ability and gene silencing efficacy. Experimental results demonstrate that the NP platform with larger size (NP-180) shows higher cellular uptake and efficient CCL-18 silencing in macrophages, leading to efficient inhibition of the breast cancer cell migration. Notably, this NP platform may passively target the 
tumor tissues via the EPR effect. However, the NP size is very large $(\sim 180 \mathrm{~nm})$ and will affect the therapeutic effect if used for in vivo regulation of macrophage behaviors. We are currently optimizing the NP formulation and small size NPs will be developed in the future for in vivo regulation of macrophage behaviors and cancer treatment.

\section{AUTHOR CONTRIBUTIONS}

SL, HY, YY, and XX conceived and designed the experiments. SL, JZ, WW, QL, and JC performed the experiments. SL, PS, XX, HY, and YY analyzed the data and co-wrote the paper.

\section{REFERENCES}

Aouadi, M., Tesz, G. J., Nicoloro, S. M., Wang, M., Chouinard, M., Soto, E., et al. (2009). Orally delivered siRNA targeting macrophage Map4k4 suppresses systemic inflammation. Nature 458, 1180-1184. doi: 10.1038/nature07774

Bronte, V., and Murray, P. J. (2015). Understanding local macrophage phenotypes in disease: modulating macrophage function to treat cancer. Nat. Med. 21, 117-119. doi: 10.1038/nm.3794

Burnett, J. C., and Rossi, J. J. (2012). RNA-based therapeutics: current progress and future prospects. Chem. Biol. 19, 60-71. doi: 10.1016/j.chembiol.2011.12.008

Caillou, B., Talbot, M., Weyemi, U., Pioche-Durieu, C., Al Ghuzlan, A., Bidart, J. M., et al. (2011). Tumor-associated macrophages (TAMs) form an interconnected cellular supportive network in anaplastic thyroid carcinoma. PLoS One 6:e22567. doi: 10.1371/journal.pone.0022567

Champion, J. A., Walker, A., and Mitragotri, S. (2008). Role of particle size in phagocytosis of polymeric microspheres. Pharm. Res. 25, 1815-1821. doi: 10. 1007/s11095-008-9562-y

Chen, J., Ding, J., Wang, Y., Cheng, J., Ji, S., Zhuang, X., et al. (2017). Sequentially responsive shell-stacked nanoparticles for deep penetration into solid tumors. Adv. Mater 29:1701170. doi: 10.1002/adma.201701170

Chen, J., Yao, Y., Gong, C., Yu, F., Su, S., Chen, J., et al. (2011). CCL18 from tumorassociated macrophages promotes breast cancer metastasis via PITPNM3. Cancer Cell 19, 541-555. doi: 10.1016/j.ccr.2011.02.006

Cheng, W., Nie, J., Gao, N., Liu, G., Tao, W., Xiao, X., et al. (2017). A Multifunctional nanoplatform against multidrug resistant cancer: merging the best of targeted chemo/gene/photothermal therapy. Adv. Funct. Mater 27:1704135. doi: 10.1002/adfm.201704135

Ginhoux, F., Schultze, J. L., Murray, P. J., Ochando, J., and Biswas, S. K. (2015). New insights into the multidimensional concept of macrophage ontogeny, activation and function. Nat. Immunol. 17:34. doi: 10.1038/ni.3324

Gordon, S., and Martinez, F. O. (2010). Alternative activation of macrophages: mechanism and functions. Immunity 32, 593-604. doi: 10.1016/j.immuni.2010. 05.007

Kanasty, R., Dorkin, J. R., Vegas, A., and Anderson, D. (2013). Delivery materials for siRNA therapeutics. Nat. Mater 12, 967-977. doi: 10.1038/nmat3765

Kortylewski, M., Swiderski, P., Herrmann, A., Wang, L., Kowolik, C., Kujawski, M., et al. (2009). In vivo delivery of siRNA to immune cells by conjugation to a TLR9 agonist enhances antitumor immune responses. Nat. Biotechnol. 27, 925-932. doi: 10.1038/nbt.1564

Liang, S., Yang, X.-Z., Du, X.-J., Wang, H.-X., Li, H.-J., Liu, W.-W., et al. (2015). Optimizing the size of micellar nanoparticles for efficient siRNA delivery. $A d v$. Funct. Mater 25, 4778-4787. doi: 10.1002/adfm.201501548

Liu, S., Pan, J., Liu, J., Ma, Y., Qiu, F., Mei, L., et al. (2018). Dynamically PEGylated and borate-coordination-polymer-coated polydopamine nanoparticles for synergetic tumor-targeted, chemo-photothermal combination therapy. Small 14:1703968. doi: 10.1002/smll.201703968

Mantovani, A., Allavena, P., Sica, A., and Balkwill, F. (2008). Cancer-related inflammation. Nature 454, 436-444. doi: 10.1038/nature07205

Mao, C. Q., Xiong, M. H., Liu, Y., Shen, S., Du, X. J., Yang, X. Z., et al. (2014). Synthetic lethal therapy for KRAS mutant non-small-cell lung carcinoma with

\section{FUNDING}

This work was supported by grants from the Natural Science Foundation of China (81874226, 81272897, 81772837, 81372819, and 81702618), the Thousand Talents Program for Distinguished Young Scholars, the National Key Research and Development Program of China (2017YFA0106300 and 2016YFC1302300), the Guangdong Science and Technology Department (2014A050503029, 2015B050501004, and 2016B030229004), the Natural Science Foundation of Guangdong Province (2017A030313828), and the funding from Guangzhou Science and Technology Bureau (201704020131).

nanoparticle-mediated CDK4 siRNA delivery. Mol. Ther. 22, 964-973. doi: 10.1038/mt.2014.18

McAllister, S. S., and Weinberg, R. A. (2014). The tumour-induced systemic environment as a critical regulator of cancer progression and metastasis. Nat. Cell Biol. 16, 717-727. doi: 10.1038/ncb3015

Melnikova, I. (2007). RNA-based therapies. Nat. Rev. Drug Discov. 6:863. doi: $10.1038 / \mathrm{nrd} 2443$

Nie, Y., Chen, J., Huang, D., Yao, Y., Chen, J., Ding, L., et al. (2017). Tumorassociated macrophages promote malignant progression of breast phyllodes tumors by inducing myofibroblast differentiation. Cancer Res. 77, 3605-3618. doi: 10.1158/0008-5472.can-16-2709

Noy, R., and Pollard, J. W. (2014). Tumor-associated macrophages: from mechanisms to therapy. Immunity 41, 49-61. doi: 10.1016/j.immuni.2014. 06.010

Ostuni, R., Kratochvill, F., Murray, P. J., and Natoli, G. (2015). Macrophages and cancer: from mechanisms to therapeutic implications. Trends Immunol. 36, 229-239. doi: 10.1016/j.it.2015.02.004

Parayath, N. N., Parikh, A., and Amiji, M. M. (2018). Repolarization of tumorassociated macrophages in a genetically engineered nonsmall cell lung cancer model by intraperitoneal administration of hyaluronic acid-based nanoparticles encapsulating microRNA-125b. Nano Lett. 18, 3571-3579. doi: 10.1021/acs. nanolett.8b00689

Qian, B.-Z., and Pollard, J. W. (2010). Macrophage diversity enhances tumor progression and metastasis. Cell 141, 39-51. doi: 10.1016/j.cell.2010.03.014

Saw, P. E., Zhang, A., Nie, Y., Zhang, L., Xu, Y., and Xu, X. (2018). Tumorassociated fibronectin targeted liposomal nanoplatform for cyclophilin a siRNA delivery and targeted malignant glioblastoma therapy. Front. Pharmacol. 9:1194. doi: 10.3389/fphar.2018.01194

Shi, J., Kantoff, P. W., Wooster, R., and Farokhzad, O. C. (2017). Cancer nanomedicine: progress, challenges and opportunities. Nat. Rev. Cancer 17, 20-37. doi: 10.1038/nrc.2016.108

Sica, A., and Mantovani, A. (2012). Macrophage plasticity and polarization: in vivo veritas. J. Clin. Invest. 122, 787-795. doi: 10.1172/JCI59643

Su, S., Liu, Q., Chen, J., Chen, J., Chen, F., He, C., et al. (2014). A positive feedback loop between mesenchymal-like cancer cells and macrophages is essential to breast cancer metastasis. Cancer Cell 25, 605-620. doi: 10.1016/j.ccr.2014. 03.021

Sun, T. M., Du, J. Z., Yan, L. F., Mao, H. Q., and Wang, J. (2008). Selfassembled biodegradable micellar nanoparticles of amphiphilic and cationic block copolymer for siRNA delivery. Biomaterials 29, 4348-4355. doi: 10.1016/ j.biomaterials.2008.07.036

Tabata, Y., and Ikada, Y. (1988). Effect of the size and surface charge of polymer microspheres on their phagocytosis by macrophage. Biomaterials 9, 356-362. doi: 10.1016/0142-9612(88)90033-6

Tseng, Y. C., Mozumdar, S., and Huang, L. (2009). Lipid-based systemic delivery of siRNA. Adv. Drug Deliv. Rev. 61, 721-731. doi: 10.1016/j.addr.2009.03.003

Wang, H. X., Xiong, M. H., Wang, Y. C., Zhu, J., and Wang, J. (2013). $\mathrm{N}$-acetylgalactosamine functionalized mixed micellar nanoparticles for targeted delivery of siRNA to liver. J. Control. Release 166, 106-114. doi: 10.1016/j. jconrel.2012.12.017 
Wang, Y.-C., Shen, S.-Y., Wu, Q.-P., Chen, D.-P., Wang, J., Steinhoff, G., et al. (2006). block copolymerization of $\varepsilon$-caprolactone and 2-methoxyethyl ethylene phosphate initiated by aluminum isopropoxide: synthesis, characterization, and kinetics. Macromolecules 39, 8992-8998. doi: 10.1021/ma061821c

Whitehead, K. A., Langer, R., and Anderson, D. G. (2009). Knocking down barriers: advances in siRNA delivery. Nat. Rev. Drug Discov. 8, 129-138. doi: 10.1038/ $\operatorname{nrd} 2742$

Xiao, H., Yan, L., Dempsey, E. M., Song, W., Qi, R., Li, W., et al. (2018). Recent progress in polymer-based platinum drug delivery systems. Prog. Polym. Sci. 87, 70-106. doi: 10.1016/j.progpolymsci.2018.07.004

Xu, X., Saw, P. E., Tao, W., Li, Y., Ji, X., Yu, M., et al. (2017a). tumor microenvironment-responsive multistaged nanoplatform for systemic RNAi and cancer therapy. Nano Lett. 17, 4427-4435. doi: 10.1021/acs.nanolett. $7 \mathrm{~b} 01571$

Xu, X., Wu, J., Liu, Y., Saw, P. E., Tao, W., Yu, M., et al. (2017b). multifunctional envelope-type siRNA delivery nanoparticle platform for prostate cancer therapy. ACS Nano 11, 2618-2627. doi: 10.1021/acsnano.6b07195

Xu, X., Wu, J., Liu, Y., Yu, M., Zhao, L., Zhu, X., et al. (2016). Ultra-pH-responsive and tumor-penetrating nanoplatform for targeted siRNA delivery with robust anti-cancer efficacy. Angew. Chem. Int. Ed. 55, 7091-7094. doi: 10.1002/anie. 201601273

Yu, S. S., Lau, C. M., Barham, W. J., Onishko, H. M., Nelson, C. E., Li, H., et al. (2013). Macrophage-specific RNA interference targeting via "click", mannosylated polymeric micelles. Mol. Pharm. 10, 975-987. doi: 10.1021/ mp300434e

Zeng, X., Liu, G., Tao, W., Ma, Y., Zhang, X., He, F., et al. (2017). A drug-self-gated mesoporous antitumor nanoplatform based on $\mathrm{pH}$-sensitive dynamic covalent bond. Adv. Funct. Mater 27:1605985. doi: 10.1002/adfm.201605985

Zhang, Y., Satterlee, A., and Huang, L. (2012). In vivo gene delivery by nonviral vectors: overcoming hurdles? Mol. Ther. 20, 1298-1304. doi: 10.1038/mt.2012. 79

Zuckerman, J. E., and Davis, M. E. (2015). Clinical experiences with systemically administered siRNA-based therapeutics in cancer. Nat. Rev. Drug Discov. 14, 843-856. doi: 10.1038/nrd4685

Conflict of Interest Statement: The authors declare that the research was conducted in the absence of any commercial or financial relationships that could be construed as a potential conflict of interest.

Copyright (c) 2018 Liang, Zheng, Wu, Li, Saw, Chen, Xu, Yao and Yao. This is an open-access article distributed under the terms of the Creative Commons Attribution License (CC BY). The use, distribution or reproduction in other forums is permitted, provided the original author(s) and the copyright owner(s) are credited and that the original publication in this journal is cited, in accordance with accepted academic practice. No use, distribution or reproduction is permitted which does not comply with these terms. 